\title{
A Modified Kelvin Model for Thermal Performance Simulation of High Mechanical Property Open-Cell Metal Foams
}

\author{
Chuan Zhang*, Feng Zhu, Houssem Badreddine, Xiaolu Gong* \\ LASMIS, Charles Delaunay Institute, University of Technology of Troyes (UTT), UMR CNRS 6281, Troyes, France \\ Email: ${ }^{*}$ chuan.zhang@utt.f, ${ }^{*}$ gong@utt.fr
}

Received 29 April 2015; accepted 24 June 2015; published 1 July 2015

\begin{abstract}
This paper proposes a modified Kelvin model for high mechanical property open-cell metal foams and investigates its application in thermal simulations. The thermal conductivity is simulated based on the steady state method and the results are consistent with experimental values. The melting process of phase change materials (PCMs) in Kelvin model and its modified model is numerically investigated under a temperature constant heat resource. By detecting the temperature variations, it shows that the metal foam greatly improves the heat transfer in energy storage systems. Besides, the comparison of the melting process in two foam models indicates that the systems based on high mechanical property metal foams have a shorter melting time. The melting process of paraffin in modified Kelvin metal foam models with three different porosities $65 \%, 70 \%$ and $75 \%$ ) are numerically analyzed and compared.
\end{abstract}

\section{Keywords}

Simulation, Metal Foam, Phase Change Materials (PCMs), Melting, Heat Transfer

\section{Introduction}

Phase change materials (PCMs) can be widely used in many thermal management systems and thermal energy storage systems due to their large latent heat. However, PCMs always suffer from the low thermal conductivities, which reduce greatly the energy storage and release efficiency. To solve this problem, many researchers have added many kinds of high thermal conductivity materials in PCMs to improve the energy transfer efficiency of systems, such as metal fins, metal meshes and metal foam structures. Among these materials, open-cell metal foams have been widely investigated because of its high surface area to volume ratio which can greatly increase the energy storage efficiency. So far, the researchers focused often on high porosity metal foams [1] [2]. However, the low mechanical properties of these metal foams can't satisfy the requirements of many applications, such as energy efficient buildings [3]. Therefore, it is needed to reduce their porosities to produce metal foams

\footnotetext{
${ }^{*}$ Corresponding authors.
}

How to cite this paper: Zhang, C., Zhu, F., Badreddine, H. and Gong, X.L. (2015) A Modified Kelvin Model for Thermal Performance Simulation of High Mechanical Property Open-Cell Metal Foams. Journal of Materials Science and Chemical Engineering, 3, 113-118. http://dx.doi.org/10.4236/msce.2015.37015 
with high mechanical property. However, few works have concentrated on the heat transfer in low porosity metal foams.

In this paper, a model of the high mechanical property open-cell metal foam is proposed and validated by comparing with the experimental results. The melting processes of paraffin in two models are both simulated and the results are compared and discussed.

\section{Modeling Method}

\subsection{Modified Kelvin Model for High Mechanical Property Open-Cell Metal Foam}

In order to describe the microstructure of metal foams, many models have been proposed. The spherical cell model, the cubical cell model, the dodecahedron model and the Kelvin (tetrakaidecahedron) model are widely used to represent the porous forms of metal foams. Among these models, the Kelvin model corresponds mostly to the real structure of metal foams whose porosity is more than $90 \%$. However, the high mechanical property aluminum foam prepared by infiltration casting method, whose porosity is $50 \%-70 \%$, has a different structure compared to high porosity foams, as shown in Figure 1. Because of the casting fabrication method, the aluminum has complex structure instead of the simple fiber form, and its pore form depends on the preform structure. In this case, the modified Kelvin model is proposed to represent the high mechanical property metal foam, as shown in Figure 2. The modified Kelvin model consists of a tetrakaidecahedron part and a solid porous unit, which represent respectively the pore and the aluminum. The porosity of the model could be adjusted by changing the square area in the tetrakaidecahedron.

\subsection{Simulation of the Thermal Conductivity}

For high mechanical property metal foams, the thermal conductivity is very important because the heat conduct is the main form of the heat transfer in the foam/PCM composite. The investigate method of foam thermal

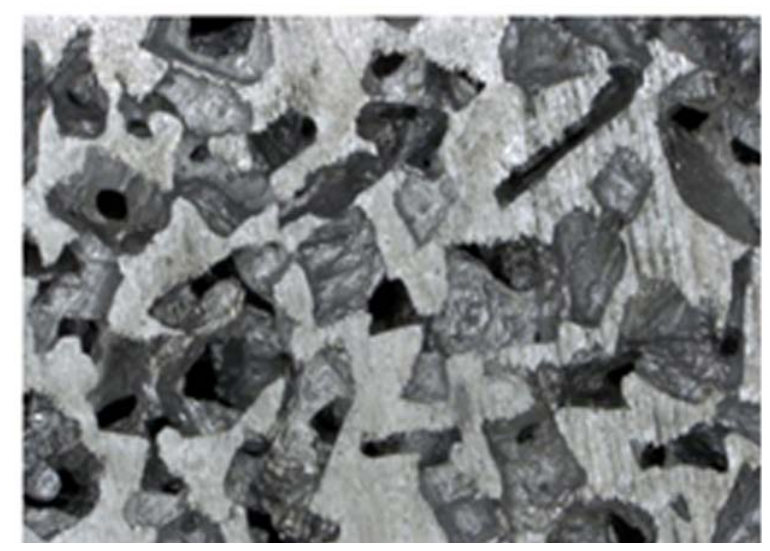

Figure 1. SEM image of the low porosity aluminum foam structure.

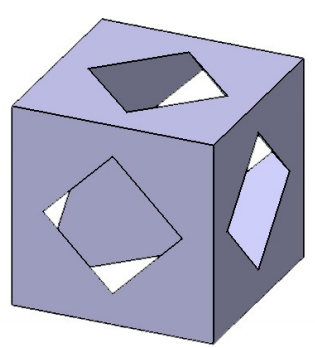

(a)

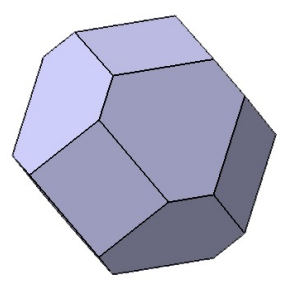

(b)

Figure 2. Modified Kelvin model (65\%), (a) metal part and (b) pore part (tetrakaidecahedron). 
conductivity is according to the reference [4]. The model is placed in the condition that the temperature gradient is $1 \mathrm{~K}$. The heat flux density will approach a constant number when the system arrives at steady state. Therefore, by using the Fourier law, the thermal conductivity could be obtained. The model grid used in this analysis is the tetrahedral mesh. In order to confirm the grid number, the model with different grid numbers is simulated at the condition discussed above. The heat flux density of middle surface is detected during the process. Figure 3 shows the heat flux density as a function of grid number. It obvious that the value of the heat flux become steady as the grid number increasing. As a result, considering both the precision and calculate time, the grid number is set to 785,257 .

\subsection{Validation of the Kelvin Model}

To verify the modified Kelvin model, the thermal conductivities of metal foams are simulated and compared with the experimental results [5]. The aluminum alloys is A356 and their porosities are from 57\% - 69\%. Figure 4 presents the comparison results. It is obvious that the thermal conductivity decreases as the foam porosity increases. This phenomena is due to the huge different of the thermal conductivity of the aluminum and air. So, the increase of the air volume could lead to the thermal conductivity decrease. Besides, in the compare results, the simulation results are consistent with the experimental ones. Therefore, the modified Kelvin model could be applied in thermal simulations of high mechanical propertymetal foams.

\subsection{Finite Element Model}

In our work, the finite element model consists of four units which represent the thickness of a layer energy

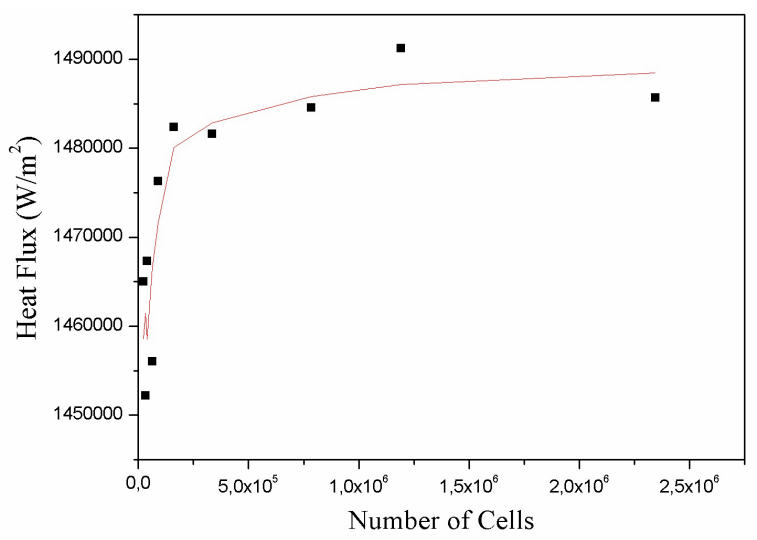

Figure 3. Heat flux as function of number of cells.

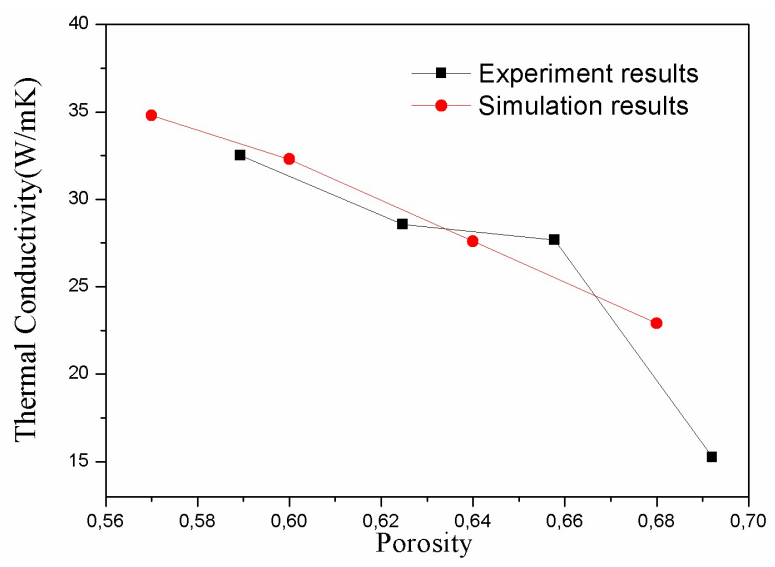

Figure 4. Comparison of experimental and numerical thermal conductivities. 
storage system, as shown in Figure 5. The simulations are achieved by ANSYS Fluent ${ }^{\circledR}$ and the heat conduction is solved by the simple algorithm according to the energy conservation equation. The mesh type is tetrahedral and its size is uniform. The dimension of each unit is one millimeter. The aluminum foam is made of AS7G, which is filled by paraffin. Their thermal properties are based on reference [6] [7]. The temperature of the heat surface is $350 \mathrm{~K}$ which is higher than the paraffin melting temperature ( $321 \mathrm{~K}-335 \mathrm{~K}$ ). The other surfaces are insulated. The initial temperature of models is $300 \mathrm{~K}$ which makes paraffin in solid state.

\section{Results and Discussions}

\subsection{Melting Process}

To observe the paraffin melting process, a cross section in the middle of model is selected as observation surface for each model, which is shown in Figure 6. The results of high mechanical property model indicate that the paraffin melts from the interfaces to the center. A similar phenomenon can be observed in the high porosity model, the paraffin begins to melt at the interfaces. Moreover, the temperature of aluminum is always higher than the paraffin. This phenomenon could be explained that the paraffin is not only heated by heat surface, but also heated by the interfaces because of the high thermal conductivity of aluminum.

In order to compare the melting process in different units, four representative points (F1, F2, F4 and F5) of 70\% porosity model and four points (P1, P2, P3 and P4) of pure paraffin are chosen as observation points who are located in the center of each cube unit. Their temperature curves are shown in Figure 7. The point F4 which is farthest from the heat surface is heated in the beginning. The temperature curves of all four points of $70 \%$

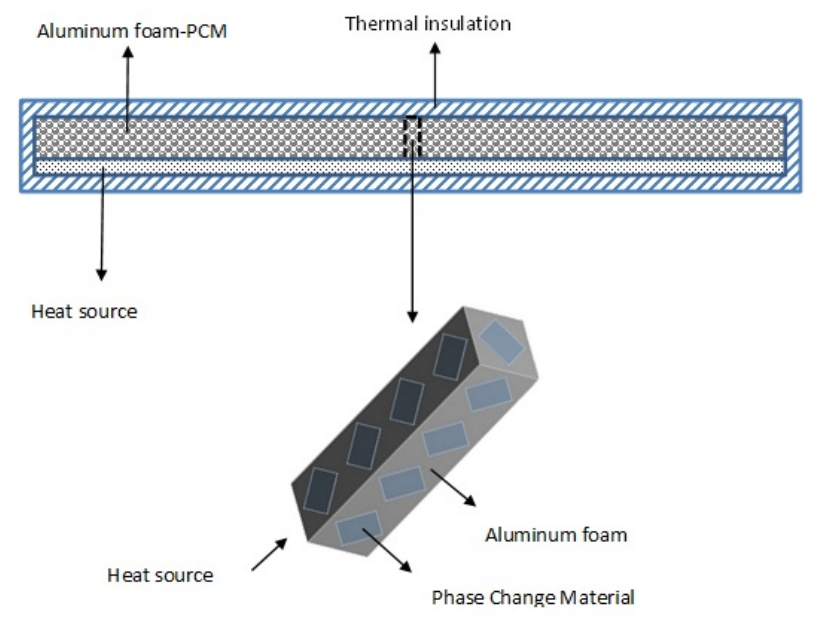

Figure 5. Layer energy storage system and his representative model.

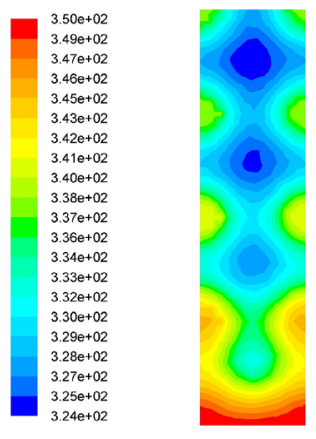

(a)

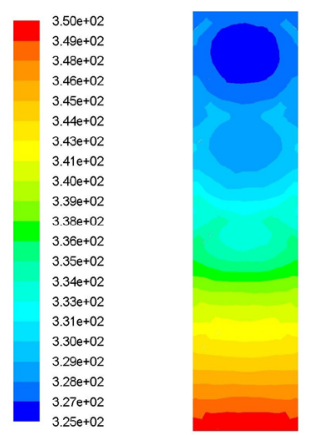

(b)

Figure 6. Temperature fields of two models, (a) modified Kelvin model (65\% porosity) and (b) Kelvin model (96\% porosity). 
model are very similar. However, the curves of pure paraffin are different. The point P4 which located in thesame position as F4 is much more difficult to be heated. The complete melting times of $70 \%$ model and pure paraffin model are 5.8 and 337 seconds. The comparison shows that the aluminum can greatly enhance the melting rate of systems, thereby reducing the time of energy storage and release.

\subsection{Comparison of Different Structural Models}

For each type model, modified Kelvin model (70\% porosity) and Kelvin model (96\% porosity) are selected to analyze the effect of structure. Two liquid fraction curves of paraffin are shown in Figure 8. The paraffin in modified Kelvin model melts completely in 5.8 seconds which is much shorter than the melting process of high porosity model. The melting rate of modified Kelvin model is largely higher than the one of Kelvin model. Firstly, the increased volume of metal helps to accelerate the heat transfer. Secondly, the high mechanical property foams have more contact surface which can heat efficiently the paraffin, so that the paraffin melts faster.

\subsection{Effect of Porosity}

Because high mechanical foams have a better performance in energy storage systems than high porosity foams, three different porosities (65\%, 70\% and 75\%) of high mechanical property foams are analyzed. Their liquid fraction curves of different porosities are shown in Figure 9. From these three curves, the different melting times can be easily observed. The melting time increases with the increase of foam porosity. Therefore, for lower foam porosity, the extra aluminum helps transfer the heat from heat resource to the paraffin, but the heat storage amount reduces as a consequence of the decreased volume fraction of paraffin.

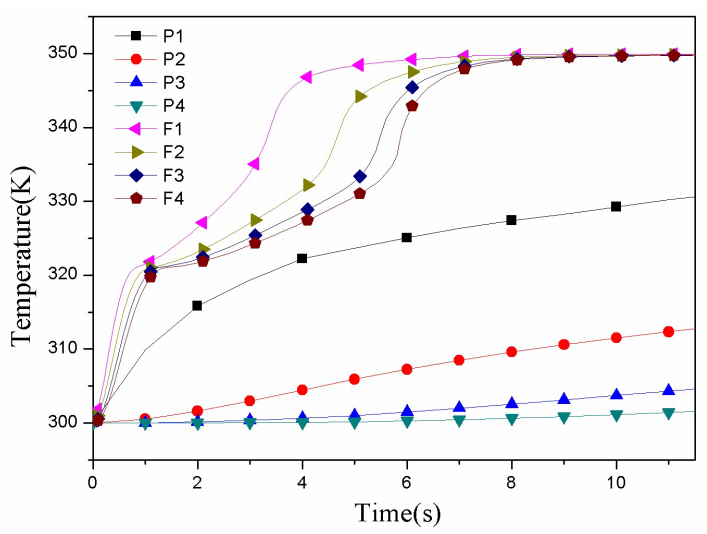

Figure 7. Temperature variations of eight center points of modified model and pure paraffin.

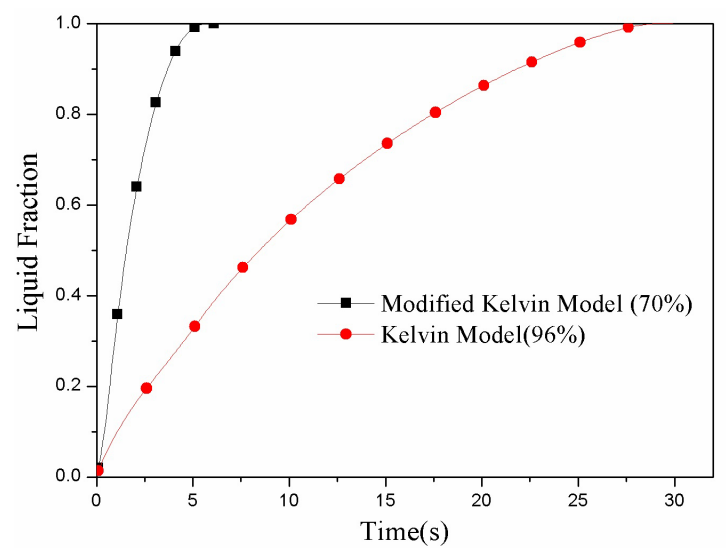

Figure 8. Comparison of liquid fraction between modified model and Kelvin model. 


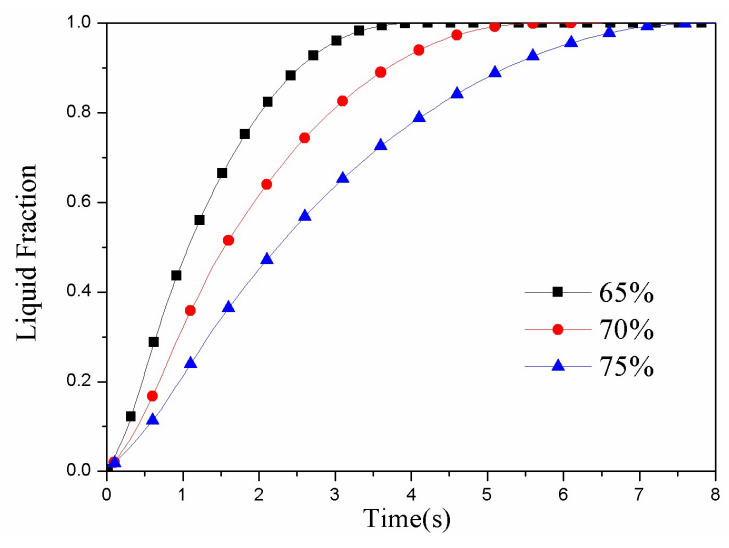

Figure 9. Liquid fraction variations of paraffin in three different porosity models of modified Kelvin model.

\section{Conclusion}

In this research, a modified Kelvin model is developed to represent high mechanical property metal foams by verifying with experimental results. The melting processes of paraffin in high mechanical property and high porosity metal foams are studied. Metal foam plays an important role in the melting process of PCMs by separating PCMs in very small pieces and heating separately them, thereby reducing largely the melting time. For high mechanical property metal foams, even though the heat storage amount of systems is less, the melting process becomes much shorter than high porosity metal foams. As a result, the energy storage systems which are made by high mechanical property metal foams could store effectively the heat in a very short time.

\section{Acknowledgements}

The authors gratefully acknowledge the financial supports for this work: the Chinese Scholarship Council (CSC) and the General Council of Champagne-Ardenne France in the project Effi-SiEMCE.

\section{References}

[1] Baby, R. and Balaji, C. (2013) Experimental Investigations on Thermal Performance Enhancement and Effect of Orientation on Porous Matrix Filled PCM Based Heat Sink. International Communications in Heat and Mass Transfer, 46, 27-30. http://dx.doi.org/10.1016/j.icheatmasstransfer.2013.05.018

[2] Sundarram, S.S. and Li, W. (2014) The Effect of Pore Size and Porosity on Thermal Management Performance of Phase Change Material Infiltrated Microcellular Metal Foams. Applied Thermal Engineering, 64, 147-154. http://dx.doi.org/10.1016/j.applthermaleng.2013.11.072

[3] Tyagi, V.V. and Buddhi, D. (2007) PCM Thermal Storage in Buildings: A State of Art. Renewable and Sustainable Energy Reviews, 11, 1146-1166. http://dx.doi.org/10.1016/j.rser.2005.10.002

[4] Moeini Sedeh, M. and Khodadadi, J.M. (2013) Thermal Conductivity Improvement of Phase Change Materials/Graphite Foam Composites. Carbon, 60, 117-128. http://dx.doi.org/10.1016/j.carbon.2013.04.004

[5] Song, J.Z. and He, S.Y. (2008) The Heat Transfer Performance of Porous Aluminum Foam. Jiangsu Metallurgy, 36, 28-30. http://www.cqvip.com/qk/95422x/200802/27218646.html

[6] Zhang, J., Zhang, D.Q., Wu, P.W., Wang, G., Li, F. and Dai, P.L. (2014) Numerical Simulation Research of Investment Casting for TiB2/A356 Aluminum Base Composite. Rare Metal Materials and Engineering, 43, 47-51. http://dx.doi.org/10.1016/S1875-5372(14)60050-3

[7] Liu, Z., Yao, Y. and Wu, H. (2013) Numerical Modeling for Solid-Liquid Phase Change Phenomena in Porous Media: Shell-and-Tube Type Latent Heat Thermal Energy Storage. Applied Energy, 112, 1222-1232.

http://dx.doi.org/10.1016/j.apenergy.2013.02.022 